\section{Chlorine-Free Synthesis for Green Chemistry}

\author{
F. Arico, A. Vavasori, Z. Liu, and T. Jiang (eds.) \\ Special Topic Issue, Pure and Applied Chemistry, \\ 2012, Vol, 84, No. 3, pp. 411-860
}

A special topic issue of Pure and Applied Chemistry published in March 2012 and titled "Chlorine-free synthesis for Green Chemistry" explores the restriction, or preferably prevention, of the use of halogenated compounds, whenever feasible, through the assembly and reporting of already identified information. Innovative synthetic pathways using clearly identified production drivers (e.g., environmental and health impact, energy consumption, economical feasibility, etc.) have been elucidated. In past decades, scientific knowledge and feasible technologies were unavailable, but now there is enough expertise to pursue discontinuation of hazardous and toxic reagents. This PAC Special Topic issue presents a collection of useful and industrially relevant examples for alternatives to chlorine in synthesis.

\section{Overview}

\section{by Pletro Tundo*}

In the last 20 years, chemists have put enormous effort into designing chemicals with various applications ranging from medicines and cosmetics to materials and molecular machines. However, for the most part, their work demonstrated a quite surprising lack of interest in taking hazards into consideration in the design process. The goal was often to design substances that were robust and could last as long as possible. This philosophy has resulted in a legacy of persistent toxic and bio-accumulative substances and lingering toxic waste sites. Nowadays, it is known that it is more desirable to avoid substances that persist indefinitely in the environment or in a landfill, and to replace them with substances designed to degrade after use. Polymeric materials, for instance, should have no negative effect on the environment during their production, utilization, or disposal. Therefore, the

\footnotetext{
- Department of Environmental Sciences, Ca' Foscari University of Venice, Venice, Italy; E-mail: tundop@unive.it ; reproduced in part from PAC 84(3), pp. 411-423, 2012; http://dx.doi.org/10.1351/ PAC-CON-12-02-02.
}

design of safer chemicals cannot be limited to hazards associated with the manufacture and use of the chemical, but also to its disposal (i.e., its full life cycle).

Among halogens, chlorine is by far the most abundant chemical in nature and also the easiest to produce and use. This explains its predominant and seemingly irreplaceable role in the chemical industry (see figure). Five hundred companies at 650 sites around the world have the capacity to produce over $58 \times 10^{6}$ tonnes of chlorine and $62 \times 10^{6}$ tonnes of its co-product, caustic soda, per year.

For example, the European chloro-alkali industry had a production in 2009 of $9.1 \times 10^{6}$ tonnes at about 80 plants, mostly (about 95 percent) via electrolysisbased techniques (chlor-alkali industry); the sector directly employs about 40000 people in 20 European Union countries. Germany is Europe's largest chlorine producer, accounting in 2009 for 43.5 percent of European production.' Owing to their peculiar characteristics, halogens are widely used by all sectors of the chemical industry to produce solvents, catalysts, building blocks, additives, and drugs. Chlorine is a major building block in today's chemistry. More than 90 percent of pharmaceuticals contain or are manufactured using chlorine, which is also used in the production of 86 percent of crop protection chemicals. Furthermore, halogens are contained in several commodities that we all use daily as plastics (e.g., chlorine is contained in polyvinyl chloride, PVC, one of the most widely used plastic materials), solvents for dry cleaning and metal degreasing, textiles, agrochemicals and pharmaceuticals, insecticides, dyestuffs, household cleaning products, and disinfectants. Chlorine is used extensively in organic and inorganic chemistry as an oxidizing agent (i.e., water disinfectant) and as a leaving group in substitution and elimination reactions.

Chlarine compounds find use as intermediates in the production of a number of important commercial products that do not contain chlorine. Foremost examples are polycarbonates, polyurethanes, silicones, polytetrafluoroethylene, carboxymethyl cellulose, and propylene oxide.

Through a chain of chemical derivatives and relatively easily made compounds and intermediates, such molecules have used the intrinsic energy available through the use of chlorine primarily produced via electrolysis

\section{Environmental and Health Concerns}

The widespread use of halocarbons was often driven by observations that most of them were more stable 